\title{
Design of a high precision converter for multi- sensor information fusion
}

\author{
Yajun $\mathrm{Ma}^{1,2,}{ }^{*}$, Wei Xiong ${ }^{1,2}$, Zhen Wang ${ }^{1,2}$, Wenzhang $\mathrm{Li}^{1,2}$, and Jiajia $\mathrm{Xu}^{1,2}$ \\ ${ }^{1}$ Beijing Institute of Precision Mechatronics and Controls, Beijing, 100076, China \\ ${ }^{2}$ Laboratory of Aerospace Servo Actuation and Transmission, Beijing, 10076, China
}

\begin{abstract}
The converter is a measuring device and is used together with the displacement sensor. In view of the existing sensor transform device is susceptible to error and temperature drift effects acquisition accuracy is not high, we design a high precision transducer, multi-sensor information fusion for vehicle steering gear shaft angular displacement signal measurement, signal transformation and digital transmission. The converter has the characteristics of high precision, miniaturization and low cost. Multi-sensor information fusion high-precision converter adopts front-end signal amplifier circuit, following filter processing circuit and embedded software of microprocessor for online compensation to satisfy the requirements of high-precision transformation. The microcontroller is used as the main control chip to meet the requirements of 8-channel bipolar analog signal acquisition. Two 14-bit, 6-channel A/D chips are used to convert the bipolar analog signal in the range of $\pm 10 \mathrm{~V}$, and the RS422 hardware interface circuit performs digital transmission according to the time sequence specified by the central programmer. The experimental results show that the conversion accuracy of the device can reach $0.06 \%$, the digital signal transmission is stable, and it can be widely used in industrial production.
\end{abstract}

Keywords: High precision transducer, Multi-sensor information fusion, Digital transmission.

\section{Introduction}

Due to its special working environment, the aerospace industry has requirements for low power consumption, integration, light weight, and small size ${ }^{[1-5]}$. Potentiometer-type angular displacement sensors are widely used by aircraft and other products because of their high accuracy, high temperature resistance, good resistance stability, low current noise and reliable contact. Conventional aircraft generally require high flight control accuracy. In order to verify and interpret the accuracy of the aircraft's attitude angle control in flight, a high-precision angular displacement sensor and a high-precision sensor conversion device must be installed on the aircraft to measure the attitude angle. Based on the above analysis,

* Corresponding author email: mayajun3690043@163.com 
this paper designs an embedded multi-channel high-precision digital sensor conversion device, which is used to measure the angular displacement signal of the aircraft steering gear, signal conversion and digital transmission ${ }^{[6-8]}$. The high-precision conversion device belongs to a miniaturized, high-precision, multi-parameter measuring device. It is characterized by good linearity, high measurement accuracy, stable performance, relatively high reliability. It has certain market application value and promotion prospects.

\section{Design of overall scheme of multi-sensor information fusion technology}

The multi-sensor information fusion system is mainly composed of an array of distributed angular displacement sensors and an information fusion module. The four angular displacement sensors are arranged in an array to realize the measurement of angle information and temperature information. The two redundant angular displacement sensors with temperature and temperature compensation system adopt the principle of linear resistance voltage division, and the design of the multi-channel information fusion converter module solves the problem[9]. The traditional angular displacement sensor has a temperature drift problem in a high temperature environment, and realizes the real-time linear compensation function in a $200^{\circ} \mathrm{C}$ temperature environment, and the system output has good linear characteristics. The three-dimensional model diagram is shown in Figure 1

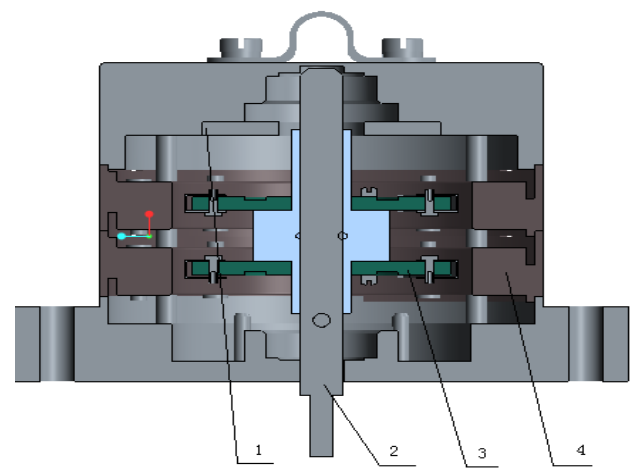

1. Temperature compensation circuit 2. Input shaft 3. Brush assembly 4. Resistance assembly

Fig.1. Structure diagram of displacement sensor with angular temperature compensation system.

In order to complete the signal processing, acquisition, transformation and transmission of the sensor. The transformation device consists of the following four parts: power conversion module, signal processing module, signal acquisition module and signal transmission module. The functional block diagram of the conversion device is shown in Figure 2. Transform device receives the upper system provides $\pm 15 \mathrm{~V}$ power supply, first by power filtering module, the adjustable three-terminal voltage regulator after transformation is $\pm 10 \mathrm{~V}$ power supply for the angular displacement sensor, transform into a $\pm 12 \mathrm{~V}$ power supply for transform device processing circuit, AD conversion circuit, power supply, finally, three-terminal voltage regulator transformation for $+5 \mathrm{~V}$ power supply for single-chip microcomputer and power interface chip. 


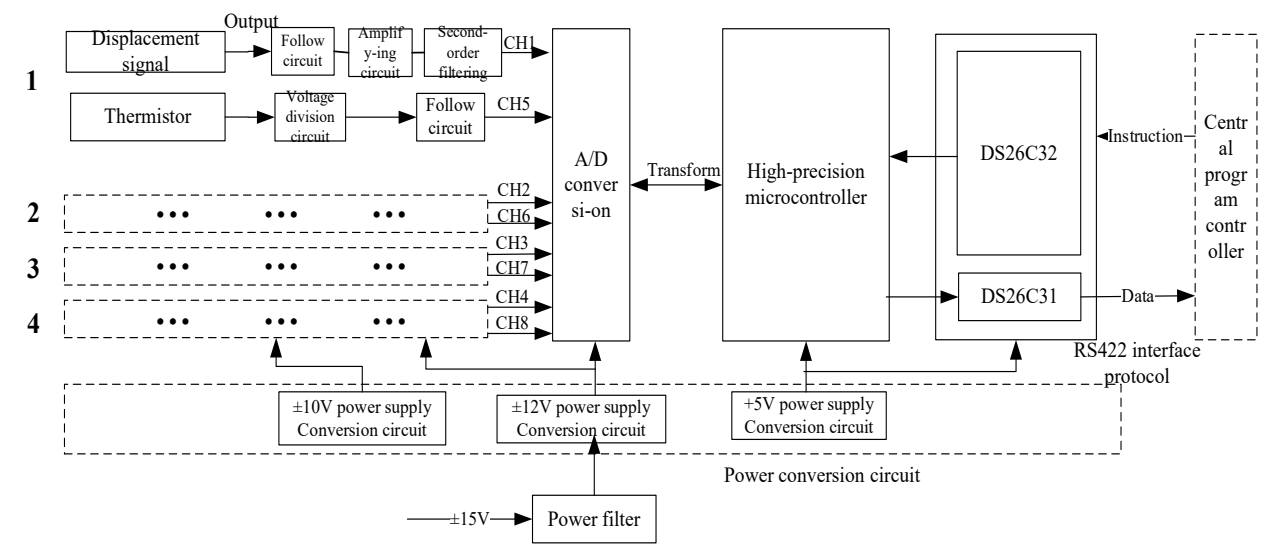

Fig. 2. Functional block diagram of multi-sensor information fusion system.

When the conversion device receives the sub-frame synchronization command from the central programmer, it starts the working program of the internal single-chip microcontroller. After receiving the word synchronization cycle command and the shift pulse command, the output signal of the angular displacement sensor is collected and converted into the digital signal is transmitted to the central programmer through the RS422 interface protocol. The software system composition is shown in Figure 3.

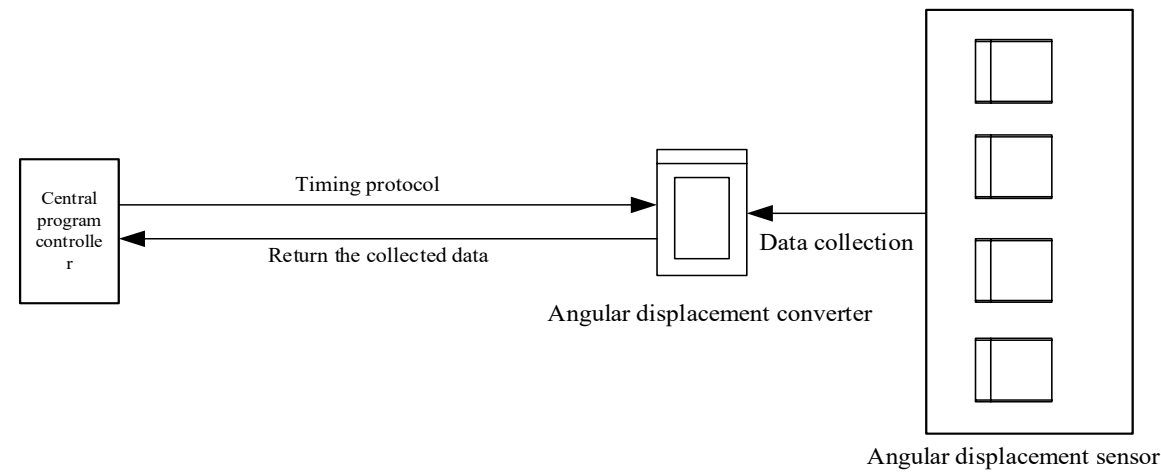

Fig. 3. Software system composition.

\section{Design of converter hardware}

The newly designed conversion device is composed of power conversion module, signal processing module, signal acquisition module and signal transmission module for hardware circuit design.

\subsection{Power conversion circuit design}

The upper system provides $\pm 15 \mathrm{~V}$ DC power supply to the sensor conversion device, and the power conversion circuit provides itself with $\pm 12 \mathrm{~V}, \pm 10 \mathrm{~V}$ and $+5 \mathrm{~V}$ power supply through DC/DC conversion.

The power conversion circuit is mainly composed of a power filter circuit, a threeterminal adjustable regulator circuit, and a three-terminal regulator circuit. The power filter 
circuit adopts an LC filter circuit to suppress spikes and transient interference on the input power of the system. The circuit is shown in Figure 4. The three-terminal adjustable voltage regulator circuit uses the LM117/LM137 chip. The chip's power supply current can reach $1.5 \mathrm{~A}$, and the voltage regulation accuracy is high. Through the selected adjustable resistance, the output conversion voltage range error is less than $0.05 \%$, which is suitable for angle accurate power supply for displacement sensor. Taking $\pm 12 \mathrm{~V}$ to $\pm 10 \mathrm{~V}$ as an example, the circuit is shown in Figure 5, in which R9, R10 and R11 are selected adjustable resistors.

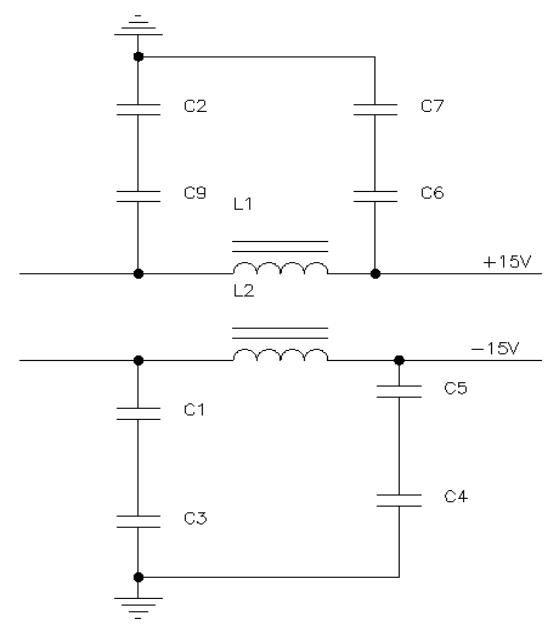

Fig. 4. Power filter circuit.

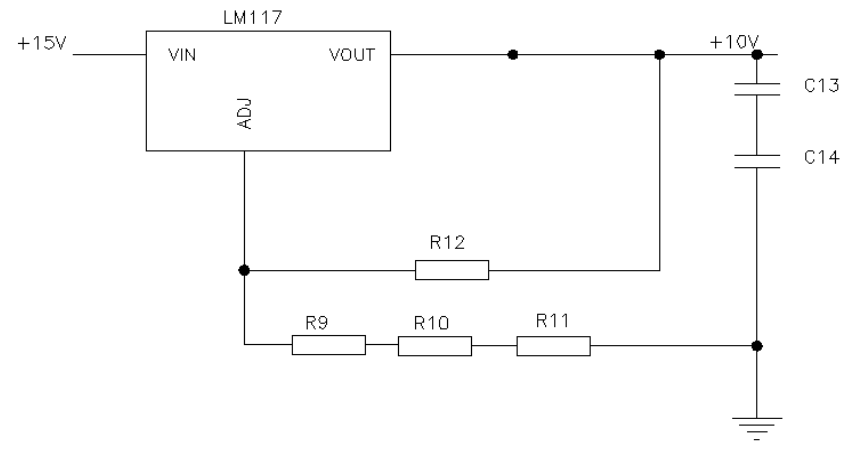

Fig. 5. Power conversion circuit.

\subsection{Signal processing circuit design}

The angular displacement sensor signal processing circuit includes temperature signal processing and displacement signal processing circuits. The temperature signal processing circuit is shown in Figure 5, including resistance divider part and voltage following part. The resistance divider circuit makes the resistance signal of thermistor turn into voltage signal output, and the following circuit performs impedance conversion and voltage following to reduce the signal attenuation caused by the output impedance. The displacement signal processing circuit is shown in Figure 6, including signal voltage following circuit, amplifier circuit and filter circuit. The following circuit, the amplifier circuit and the filter circuit use the operation amplifier chip LM124 chip, the chip contains 
four groups of completely the same form of operational amplifier, in addition to the power sharing, the four groups of operation amplifier are independent of each other. For multichannel signal acquisition, it can achieve the purpose of miniaturization. In order to prevent the interference of clutter signals, according to the requirements of the product's operating frequency, a filter circuit is designed. The filter circuit uses a Sallen-Key structure RC lowpass second-order active filter.

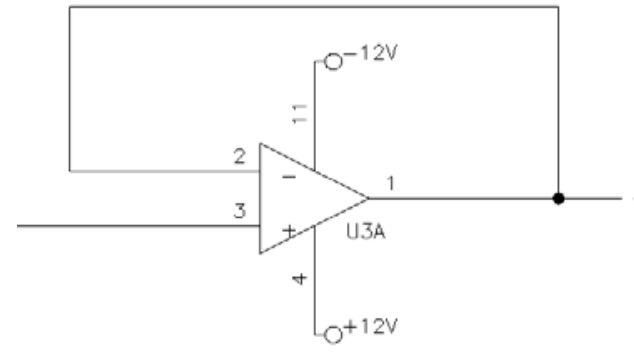

Fig. 5. Amplifier with follower circuit.

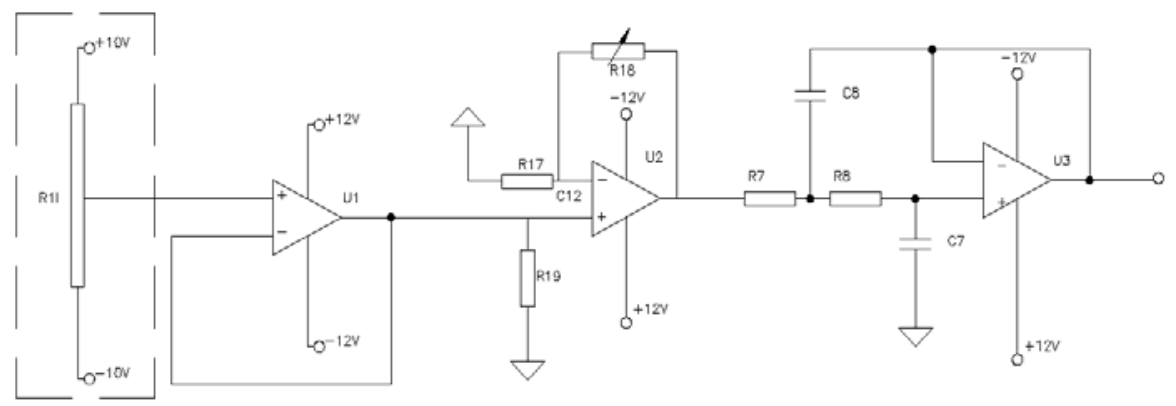

Fig. 6. Displacement signal processing circuit.

According to the required $75 \mathrm{~Hz}$ frequency, the resistance and capacitance of the filter circuit are deduced according to formula (1). The Sallen-Key structure RC low-pass filter is derived and simulated by MULTISIM software as shown in Figure 7.
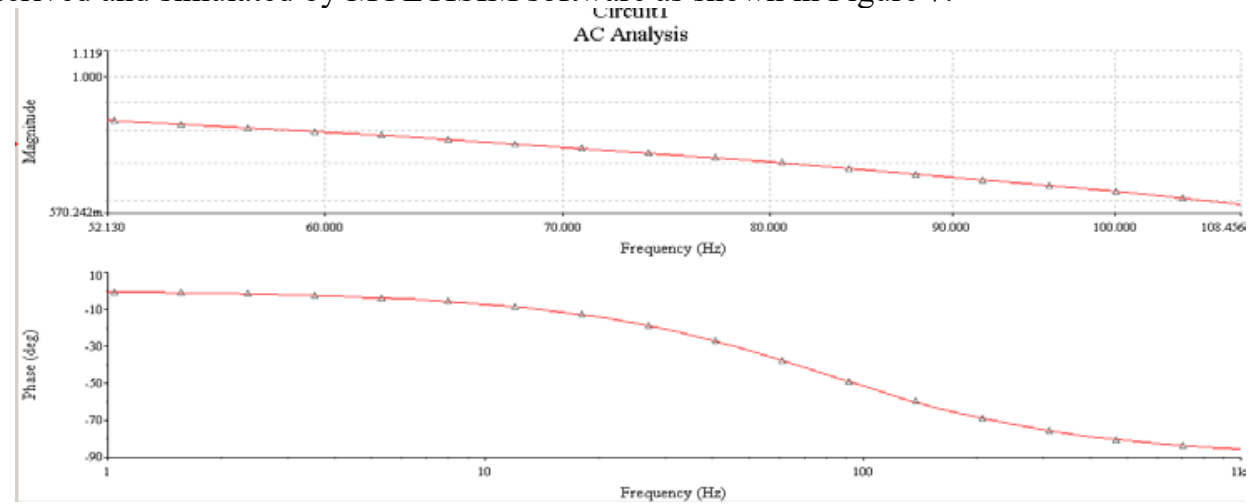

Fig. 7. MULTISIM software simulation diagram.

$$
A(S)=\frac{1}{1+w_{c} C_{7}\left(R_{7}+R_{8}\right) S+w_{C}^{2} R_{7} R_{8} C_{7} C_{8} S^{2}}
$$


According to the coefficient table of $3 \mathrm{~dB}$ Chebyshev second-order filter, we can get

$$
\begin{gathered}
a_{1}=w_{c} C_{7}\left(R_{7}+R_{8}\right)=1.0650 \\
b_{1}=w_{c}^{2} C_{7} C_{8} R_{7} R_{8}=1.9305 \\
\quad A_{0}=1 \\
C_{7}=22 n F \\
C_{8} \geq C_{7} \frac{4 b_{1}}{a_{1}^{2}}=22 n F \times \frac{4 \times 1.9305}{1.065^{2}} \cong 150 n F \\
R_{7}=\frac{1.065 \times 150 \times 10^{-9}-\sqrt{\left(1.065 \times 150 \times 10^{-9}\right)^{2}-4 \times 1.9305 \times 22 \times 10^{-9} \times 150 \times 10^{-9}}}{4 \pi \times 3 \times 10^{3} \times 22 \times 10^{-9} \times 150 \times 10^{-9}}=50.4 \mathrm{~K} \\
R_{8}=\frac{1.065 \times 150 \times 10^{-9}+\sqrt{\left(1.065 \times 150 \times 10^{-9}\right)^{2}-4 \times 1.9305 \times 22 \times 10^{-9} \times 150 \times 10^{-9}}}{4 \pi \times 3 \times 10^{3} \times 22 \times 10^{-9} \times 150 \times 10^{-9}}=52 \mathrm{~K}
\end{gathered}
$$

The values of C7, C8, R7, and R8 can be derived.

\subsection{Signal processing circuit design}

The microcontroller of the converter adopts the C8051F530A chip produced by Siliconlabs, with a built-in internal crystal oscillator of $24.5 \mathrm{MHz}$ and a processing speed of 25MIPS. The AD chip adopts imported products, and the AD7656 is selected. This chip can greatly reduce the number and volume of decoupling capacitors, reduce the scale of peripheral circuits, improve the reliability of AD conversion circuits, and can indirectly achieve the purpose of miniaturization. Two AD chips are short-circuited using the CONVST port to realize synchronous acquisition. Using the control CS chip selection, serially transmit the data into the single-chip microcomputer respectively. The signal transmission circuit uses DS26C31 and DS26C32 chips. The function of the chip circuit conforms to the function of the difference line transmitter and receiver of the universal serial bus RS422 standard, and it has strong anti-interference ability.

\subsection{Signal transmission circuit design}

The signal transmission circuit uses DS26C31 and DS26C32 chips [10]. The functions of the chip circuit conform to the difference line transmitter and receiver functions of general serial bus RS-422 standard. Differential circuit is a kind of balanced communication interface, and the transmission rate is up to $10 \mathrm{Mb} / \mathrm{s}$. It is widely used in the field of data communication.

\section{Design of converter hardware}

\subsection{Software operating environment}

The operating environment of the angular displacement converter software is shown in Table 1. 
Table 1. Software operating environment.

\begin{tabular}{lcc}
\cline { 2 - 3 } 1 & Project & Software operating environment \\
2 & Software type & Embedded Software \\
3 & Hardware environment & Converter \\
4 & Software support environment & Silicon Laboratories IDE \\
5 & Development language & Assembly programming language \\
\hline
\end{tabular}

The hardware-in-the-loop test simulation environment connection of the converter software is shown in Figure 8

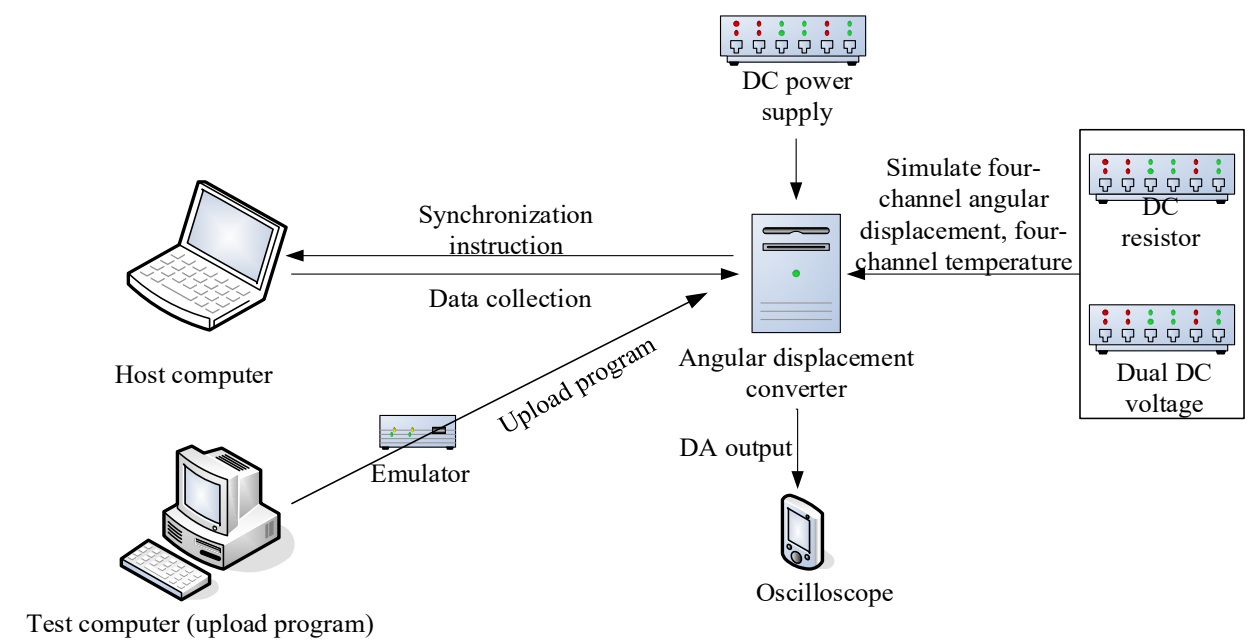

Fig. 8. Angular displacement converter software hardware-in-the-loop simulation test environment connection diagram.

\subsection{Flow chart of software design}

The converter software needs to realize the AD serial data acquisition function of 8-channel signal, the linear compensation function of 8-channel signal, and the signal transmission function according to the timing protocol. It mainly contains two parts: main program, external interrupt service program, in the external interrupt subroutine call data acquisition program, data compensation subroutine, delay subroutine, etc. The software design flow diagram is shown in Figure 9.

\subsection{Software programming language selection}

The software is programmed in assembly language. The reason for using assembly language programming is that the commonly used high-level languages such as $\mathrm{C}$ language cannot control the execution time cycle of the code, and cannot control the timing sequence of control transmission accurately. Assembly language can directly access the memory or I/O ports related to the hardware. It can completely control the generated binary code without being restricted by the compiler. It can increase the running speed, significantly reduce the execution time, and can modify the key code. Precise time sequence control is more accurate for high-speed sequence control. 


\subsection{Main functions implemented by software}

The main functions of the converter software are: initialization function, data acquisition function, linear compensation function, receiving synchronization command function, receiving control command function, data verification function, and data sending function.

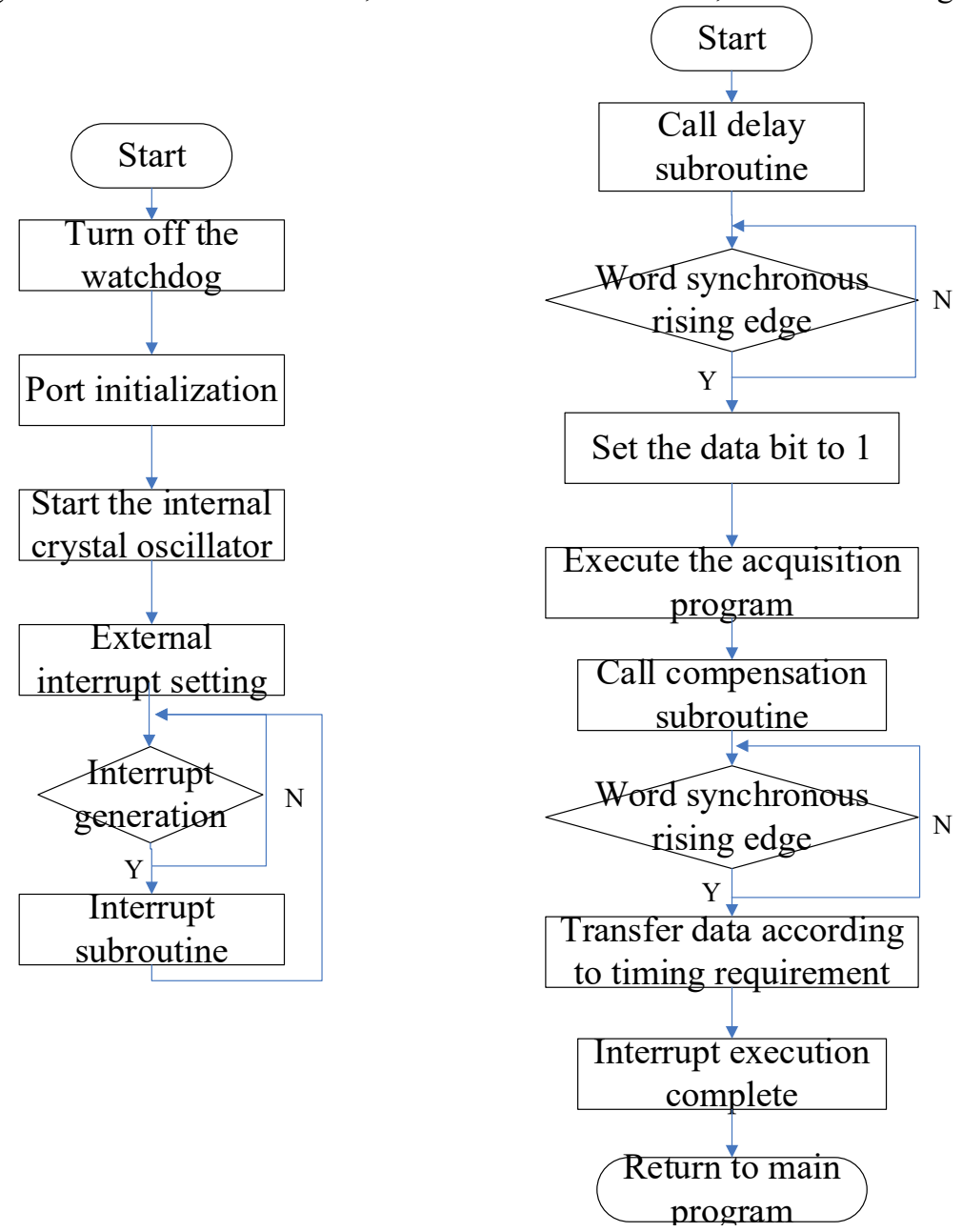

Fig. 9. Program flow chart.

\section{Converter test results and analysis}

The converter software is solidified into the MCU of the displacement converter through the burner, and the central program controller is simulated by the upper computer and the Advantech Industrial PC, and the four-way angular displacement and four-way temperature signals are simulated by the DC resistor and the dual-way DC power supply. The measurement and control software of the system is realized based on LabWindows/CVI programming [11]. The interface design of the system has complete functions and friendly human-machine interface. The conversion device is connected with two angular displacement sensors, and the signal data acquisition system is connected, as shown in Figure 10. 


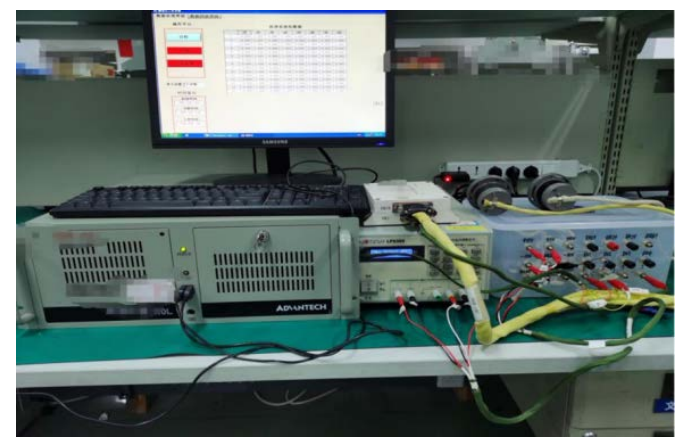

Fig. 10. Alignment equipment connection diagram.

The performance index of the angular displacement converter is mainly reflected in the test data, and the specific test result index is shown in Table 2.

Table 2. Testing data tables of angular displacement sensor signal linearity.

\begin{tabular}{|c|c|c|c|c|c|c|c|c|}
\hline $\begin{array}{l}\text { Theoret } \\
\text { ical } \\
\text { input } \\
\text { value }\end{array}$ & \multicolumn{2}{|c|}{$\begin{array}{l}\text { Displacement } \\
\text { signal I }\end{array}$} & \multicolumn{2}{|c|}{$\begin{array}{l}\text { Displacement } \\
\text { signal II }\end{array}$} & \multicolumn{2}{|c|}{$\begin{array}{l}\text { Displacement } \\
\text { signal III }\end{array}$} & \multicolumn{2}{|c|}{$\begin{array}{c}\text { Displacement } \\
\text { signal IV }\end{array}$} \\
\hline $\begin{array}{c}\text { Displac } \\
\text { ement } \\
\text { signal( } \\
\text { V) } \\
\end{array}$ & $\begin{array}{c}\text { outpu } \\
t(V)\end{array}$ & $\begin{array}{c}\text { Differenc } \\
\text { e value } \\
(\mathrm{mV})\end{array}$ & $\begin{array}{l}\text { Output } \\
\text { (V) }\end{array}$ & $\begin{array}{c}\text { Differe } \\
\text { nce } \\
\text { value } \\
(\mathrm{mV}) \\
\end{array}$ & $\begin{array}{c}\text { output( } \\
\text { V) }\end{array}$ & $\begin{array}{c}\text { differe } \\
\text { nce } \\
\text { value } \\
(\mathrm{mV}) \\
\end{array}$ & $\begin{array}{l}\text { Output } \\
\text { (V) }\end{array}$ & $\begin{array}{l}\text { differ } \\
\text { ence } \\
\text { value } \\
(\mathrm{mV}) \\
\end{array}$ \\
\hline 9.000 & 9.002 & 2 & 9.000 & 0 & 8.999 & 1 & 8.999 & 1 \\
\hline 8.000 & 8.003 & 3 & 8.000 & 0 & 8.000 & 0 & 7.998 & 2 \\
\hline 7.000 & 7.003 & 3 & 6.999 & 1 & 6.998 & 2 & 6.998 & 2 \\
\hline 6.000 & 6.002 & 2 & 5.998 & 2 & 5.999 & 1 & 5.998 & 2 \\
\hline 5.000 & 5.002 & 2 & 4.999 & 1 & 5.001 & 1 & 4.998 & 2 \\
\hline 4.000 & 4.002 & 2 & 3.998 & 2 & 3.999 & 1 & 3.997 & 3 \\
\hline 3.000 & 2.999 & 1 & 2.999 & 1 & 2.998 & 2 & 2.998 & 2 \\
\hline 2.000 & 2.001 & 1 & 1.998 & 2 & 1.999 & 1 & 1.998 & 2 \\
\hline 1.000 & 0.998 & 2 & 0.999 & 1 & 0.997 & 3 & 0.998 & 2 \\
\hline 0 & 0.001 & 1 & 0.002 & 2 & 0.001 & 1 & 0.001 & 1 \\
\hline-1.000 & -0.999 & 1 & -0.999 & 1 & -0.999 & 1 & -0.999 & 1 \\
\hline-2.000 & -1.998 & 2 & -1.998 & 2 & -1.999 & 1 & -1.998 & 2 \\
\hline-3.000 & -2.998 & 2 & -2.998 & 2 & -2.997 & 3 & -2.999 & 1 \\
\hline-4.000 & -3.999 & 1 & -4.001 & 1 & -3.999 & 1 & -3.998 & 2 \\
\hline-5.000 & -5.001 & 1 & -5.000 & 0 & -4.999 & 1 & -4.999 & 1 \\
\hline-6.000 & -6.002 & 2 & -6.001 & 1 & -5.998 & 2 & -6.000 & 0 \\
\hline-7.000 & -7.003 & 3 & -7.001 & 1 & -7.001 & 1 & -6.999 & 1 \\
\hline-8.000 & -8.002 & 2 & -8.001 & 1 & -7.999 & 1 & -7.998 & 2 \\
\hline-9.000 & -9.002 & 2 & -9.002 & 2 & -9.001 & 1 & -8.999 & 1 \\
\hline $\begin{array}{l}\text { Linear } \\
\text { ity }\end{array}$ & $\begin{array}{r}0.041 \\
\% \\
\end{array}$ & & $\begin{array}{r}0.035 \\
\%\end{array}$ & & $0.031 \%$ & & $0.032 \%$ & \\
\hline
\end{tabular}


The test results show that the acquisition accuracy of the sensor conversion device is $0.041 \%, 0.035 \%, 0.031 \%$, and $0.032 \%$ calculated according to the absolute linearity method. The product realizes the functions of multi-channel acquisition and digital transmission, and achieves the expected acquisition accuracy.

\section{Conclusion}

This paper introduces the hardware and software design of a multi-sensor information fusion high-precision converter, which realizes bipolar multi-channel acquisition, and realizes the high-precision acquisition requirements of the converter through hardware compensation and software temperature compensation. The designed converter has high data acquisition accuracy, and can provide RS422 digital signal transmission when combined with potentiometer displacement sensor, which can provide reference for the subsequent high-precision sensor digital design. As a cost-effective digital product, it can be promoted and applied in the market and has considerable economic value.

\section{References}

1. Yang X P, Feng Z H and Liu Y D 2008 Journal of Suzhou University vol 28 p 49

2. Yang M, Wang W and Wang Z 2014 Journal of Astronautic Metrology and Measurement vol $34 \mathrm{p} 162$

3. Li D J, Chen D and Ma Y J 2014 Journal of Astronautic Metrology and Measurement vol 34 p 261

4. Feng Y, Yu X H and Man Z H 2002 Automatica vol 38 p159

5. Chen C, Zhang C R and Hu T L 2019 Int J. Adv. Robot. Syst. vol 16 p 321

6. Mobayen S, Tchier F and Ragoub L 2017 Int J. Syst. Sci. vol 48 p 298

7. Levant A 2002 Int J. control. vol 76 p 29

8. Sun L, Liu Y J 2020 Mech. Syst. Sign. Proc. vol 139 p 62

9. Yassine K, Maarouf S and Khalid B 2018 Nonlinear. Dyn. vol 93 p 257

10. Zhang L Y, Liu L Z and Wang Z 2018 IET. Control. Theory. Appl vol 12 p 321

11. Utkin V I, Poznyak A S 2013 Automatica vol 49 p 39 